\title{
Video Article \\ Preparation of Acute Hippocampal Slices from Rats and Transgenic Mice for the Study of Synaptic Alterations during Aging and Amyloid Pathology
}

\author{
Diana M. Mathis ${ }^{1}$, Jennifer L. Furman ${ }^{2}$, Christopher M. Norris ${ }^{2,3}$ \\ ${ }^{1}$ Graduate Center for Gerontology, University of Kentucky College of Public Health \\ 2 Department of Molecular and Biomedical Pharmacology, University of Kentucky College of Medicine \\ ${ }^{3}$ Sanders-Brown Center on Aging, University of Kentucky College of Medicine
}

Correspondence to: Christopher M. Norris at cnorr2@uky.edu

URL: https://www.jove.com/video/2330

DOI: doi:10.3791/2330

Keywords: Neuroscience, Issue 49, aging, amyloid, hippocampal slice, synaptic plasticity, Ca2+, CA1, electrophysiology

Date Published: 3/23/2011

Citation: Mathis, D.M., Furman, J.L., Norris, C.M. Preparation of Acute Hippocampal Slices from Rats and Transgenic Mice for the Study of Synaptic Alterations during Aging and Amyloid Pathology. J. Vis. Exp. (49), e2330, doi:10.3791/2330 (2011).

\section{Abstract}

The rodent hippocampal slice preparation is perhaps the most broadly used tool for investigating mammalian synaptic function and plasticity. The hippocampus can be extracted quickly and easily from rats and mice and slices remain viable for hours in oxygenated artificial cerebrospinal fluid. Moreover, basic electrophysisologic techniques are easily applied to the investigation of synaptic function in hippocampal slices and have provided some of the best biomarkers for cognitive impairments. The hippocampal slice is especially popular for the study of synaptic plasticity mechanisms involved in learning and memory. Changes in the induction of long-term potentiation and depression (LTP and LTD) of synaptic efficacy in hippocampal slices (or lack thereof) are frequently used to describe the neurologic phenotype of cognitively-impaired animals and/ or to evaluate the mechanism of action of nootropic compounds. This article outlines the procedures we use for preparing hippocampal slices from rats and transgenic mice for the study of synaptic alterations associated with brain aging and Alzheimer's disease (AD) ${ }^{1-3}$. Use of aged rats and $A D$ model mice can present a unique set of challenges to researchers accustomed to using younger rats and/or mice in their research. Aged rats have thicker skulls and tougher connective tissue than younger rats and mice, which can delay brain extraction and/or dissection and consequently negate or exaggerate real age-differences in synaptic function and plasticity. Aging and amyloid pathology may also exacerbate hippocampal damage sustained during the dissection procedure, again complicating any inferences drawn from physiologic assessment. Here, we discuss the steps taken during the dissection procedure to minimize these problems. Examples of synaptic responses acquired in "healthy" and "unhealthy" slices from rats and mice are provided, as well as representative synaptic plasticity experiments. The possible impact of other methodological factors on synaptic function in these animal models (e.g. recording solution components, stimulation parameters) are also discussed. While the focus of this article is on the use of aged rats and transgenic mice, novices to slice physiology should find enough detail here to get started on their own studies, using a variety of rodent models.

\section{Video Link}

The video component of this article can be found at https://www.jove.com/video/2330/

\section{Preparing Ice-cold Oxygenated Artificial Cerebrospinal Fluid (ACSF)}

1. Prepare $2 \mathrm{~L}$ of "Ca ${ }^{2+}$-free" $\mathrm{ACSF}$. In a $2 \mathrm{~L}$ Erlenmeyer flask, add approximately $1.5 \mathrm{~L}$ of sterile or double-distilled $\mathrm{H}_{2} \mathrm{O}$, and begin stirring vigorously on a stir plate. Add the following ACSF components (in $\mathrm{mM}$ ): $124 \mathrm{NaCl}, 2 \mathrm{KCl}, 1.25 \mathrm{KH}_{2} \mathrm{PO}_{4}, 2 \mathrm{MgSO}_{4}, 26 \mathrm{NaHCO}_{3}$, and 10 dextrose (see Table 1). Bring to a volume of $2 \mathrm{~L}$ with distilled $\mathrm{H}_{2} \mathrm{O}$.

2. Using an aquarium bubbler and tubing attached to a $95 \% \mathrm{O}_{2} / 5 \% \mathrm{CO}_{2}$ air tank, oxygenate ACSF vigorously for approximately $20-30$ min Check the $\mathrm{pH}$, and if necessary, adjust to 7.4 using $\mathrm{NaOH}$ or $\mathrm{HCl}$.

3. Pour $750 \mathrm{~mL}$ of oxygenated $\mathrm{Ca}^{2+}$-free ACSF into a separate Erlenmeyer flask, cover the opening with parafilm, and transfer to an ultrafreezer $\left(-80^{\circ} \mathrm{C}\right)$ for $20-30 \mathrm{~min}$. This media will be used for dissection of brain and acute hippocampal slices*. Add $2 \mathrm{mM} \mathrm{CaCl}_{2}$ to the remaining $1.25 \mathrm{~L}$ volume of ACSF\#, stir well, and resume oxygenation with $95 \% \mathrm{O}_{2} / 5 \% \mathrm{CO}_{2}$. This media will be used for storing brain slices after dissections, and for perfusing slices during electrophysiologic recordings.

${ }^{*}$ Ice-cold $\mathrm{Ca}^{2+}$ free media is used to slow metabolism and minimize $\mathrm{Ca}^{2+}$-dependent excitotoxicity during dissection.

\# Changes in $\mathrm{Ca}^{2+}$ dysregulation during aging and $\mathrm{AD}$ can have a major impact on the induction of $\mathrm{Ca}^{2+}$-dependent synaptic plasticity ${ }^{4-9}$. Conflicting reports on age differences in LTD may be partly attributable to the use of different $\mathrm{ACSF} \mathrm{Ca}{ }^{2+}: \mathrm{Mg}_{2}+$ ratios during slice recordings $\left(\mathrm{see}^{2,4,10}\right)$. The importance of $\mathrm{Ca}^{2+}$ regulation and the ACSF Ca ${ }^{2+}$ level in aging studies is addressed in greater detail in the Discussion section.

4. While the $\mathrm{Ca}^{2+}$-free ACSF is freezing, prepare a holding chamber for the maintenance of brain slices before and during electrophysiologic recordings*. We use a custom macro-holding chamber that contains four individual microchambers (see Figure 2). The macrochamber is filled with sterile, oxygenated $\mathrm{H}_{2} \mathrm{O}$ and the microchambers are essentially islands that protrude above the water's surface. Within each 
microchamber, slices rest on netted insert in a shallow pool of oxygenated ACSF. Slices are not completely submerged, but sit at an interface with the humidified air. An insulated heating element within the macrochamber allows for temperature adjustment.

*Several varieties of holding chambers are also commercially available (e.g. Warner Instruments makes a submersion-style "Pre Chamber \#BSC-PC) and should be suitable for use in aging and transgenic mouse slice studies. In a pinch, slices can be maintained for several hours in a small petri dish filled with ACSF. Make a small hole in the petri lid for an oxygen delivery tube. Be careful that oxygen bubbles do not physically agitate the slices. Slices will get sufficient oxygen if the delivery tube is raised to just above the ACSF surface (gas dispersion should cause an indentation in the fluid surface).

\section{Brain Removal and Hippocampal Dissection in Aged (> 20-month-old-rats)}

1. Prepare the dissection area* next to a large sink (see Figure 1 and Table 2). Place a small animal guillotine in the sink and lay out surgical instruments and materials for brain removal and hippocampal dissection including a folded paper towel, a \#11 scalpel blade, beebee scissors, bone rongeurs, a "Hippocampus tool" (a specialized dual spatula from Fine Surgical Tools), small surgical scissors, a thin double-ended spatula, plastic Pasteur pipette, $110 \mathrm{~mm}$ diameter Whatman Filter paper, a lidded $100 \mathrm{~mm}$ glass petri dish filled with ice, and a plastic spoon. Also keep a plastic bag nearby for disposal of the carcass.

*Brain extraction should be completed as quickly as possible, so it's a good idea to mentally "walk through" the procedure and lay your instruments out in the order of use.

2. Remove $\mathrm{Ca}^{2+}$-free ACSF from the freezer. Media should be partially, not completely, frozen. Pour about $50 \mathrm{~mL}$ of ACSF into a glass beaker, cover with Parafilm, and place next to the dissection area. This media will be used to briefly store the brain once it is removed. The remaining $\mathrm{Ca}^{2+}$-free media will be used to fill the reservoir in the Vibratome for slice preparation. This media can be reoxygenated, or simply covered with parafilm and placed in the refrigerator at $4^{\circ} \mathrm{C}$.

3. Euthanize the rat using methods approved by the Institutional Animal Care and Use Committee (IACUC). Our animals are placed in a small Plexiglas chamber that is gradually filled with $100 \% \mathrm{CO}_{2}$. Loss of consciousness generally occurs within five minutes and is confirmed by the absence of reflex activity following a toe pinch.

4. Decapitate the rat immediately rostral to the first cervical vertebra and place the head on the folded paper towel. Using the \#11 scalpel, quickly make an incision in the middle of the scalp starting near the nasal bone and running caudally to the occipital bone. Be sure to cut completely through the cutaneous muscle, fully exposing the sutures on the dorsal surface of the skull.

5. Cut through the scull plates with beebee scissors. In young rats and mice, the skull can be quickly removed with the use of bone rongeurs alone. However, aged rats have thicker skulls than young adult rats and mice that can make this procedure difficult. We have found that cutting through the skull greatly facilitates bone removal with rongeurs, lessens injury to the brain, and saves precious seconds. With the rat's head firmly on the counter top, place the cutting edge of the lower sheer of the beebee scissors into the superior region of the foramen magnum at the posterior portion of the skull. Keeping the lower sheer firmly against the skull's inner surface (and away from the brain) ${ }^{\star}$, cut through the occipital plate, and then along the midline suture of the parietal plates. Proceed rostrally until you cut through the frontal skull plates.

*It is critically important that pressure is directed away from the brain to prevent inadvertent gauging.

6. Separate the occipital, parietal, and temporal skull plates from the brain. Continue holding the head firmly to the countertop for stability and leverage and slide the bottom jaw of the rongeurs under the left parietal plate maintaining pressure against the inside surface of the skull. Next, squeeze the jaws of the rongeurs together and roll your wrist upward and toward you to pull the parietal and occipital plates away from the brain. This should expose most of the dorsal surface of the left hemisphere. If necessary, use the rongeurs to remove the left frontal plate as well. Repeat this process for the other hemisphere. Once the plates are displaced, quickly inspect for any dura matter that may be attached to the temporal plates and stretched across the surface of the brain. Gently pull these away with the rongeurs or cut away with scissors*. Now, slide the upper jaw of the rongeurs between the brain and the right temporal plate, again keeping the pressure toward the skull and away from the brain. Squeeze and twist the temporal plate away from the brain. You should hear/feel a "crunch" as you do this. Repeat for the left side.

*The dura can be hard to spot, especially if the animal has been transcardially perfused with ACSF. However, if dura are not removed, they will slice through the brain (and most likely the hippocampus) like a razor when temporal plates are removed. Snipping the dura away near the temporal plates will minimize the likelihood of stabbing the brain.

7. Extract the brain*. Quickly remove the parafilm from the $\mathrm{Ca}^{2+}$-free ACSF "slushy". Now slide the broad spatula head of the Hippocampal tool between the ventral surface of the brain and the bottom skull plates until it is completely under the brain. Move the spatula laterally from side-to-side and then forward and backwards a few times to sever intact cranial nerves. Now scoop the brain out with the Hippocampal tool and submerge in $\mathrm{Ca}^{2+}$-free ACSF and cover with parafilm. Let the brain chill for about one minute. This is an opportune time to clean off the guillotine, dispose of the carcass, and reorganize the dissection area.

${ }^{*}$ For steps 2.3-2.7, speed is of the essence. We try to complete this procedure (from decapitation to brain submersion in ACSF) in 30-35 sec. In our experience, extractions taking longer than a minute appear to adversely affect hippocampal slice health, especially for aged rats. Using these procedures, we have observed no differences in extraction times between aged and young rats for our studies.

8. Extract the hippocampi. Place the Whatman filter paper on the lid of the ice-cold petri dish and dampen the paper with ACSF using the plastic transfer pipette. Retrieve the brain from the ACSF using a spoon and place on the dampened Whatman paper. Using the scalpel blade, remove the cerebellum and approximately one quarter of the rostral frontal lobes. Run the scalpel blade through the intrahemispheric fissure to completely separate the two hemispheres. Place one hemisphere back into the ACSF slushy and "stand" the other up on the dissecting stage such that the coronal plane of the frontal lobe is facing down. You should clearly be able to distinguish the brain stem and mid brain (which are white) from the overlying cortex (which is pink/grayish). Locate the superior and inferior colliculi on the midbrain; these will look like two white "knobs" and will be at the "top" of the brain in this orientation. Using the surgical scissors, gently hold the midbrain in place and slide the weighing spatula in the gap between the colliculi and the neocortex. Very gently, continue to slide the spatula down and pull the brainstem/midbrain/thalamus away revealing the inside of the lateral ventricle and the medial surface of the hippocampus. Use the sharp edge of the spatula to smoothly sever the fornix; a white fiber bundle located at the anterior/dorsal portion of the hippocampus. With the scissors, gently continue to pull the brainstem/midbrain/thalamus away without completely severing it from the rest of the brain. The cortex with hippocampus should now lay freely back from the brainstem*. Next, turn the dissecting stage so that you are looking at the medial hippocampus and overlying cortex as if it were a sagittal section (minus the thalamus). You should now see the white fimbria fibers that form a shallow hyperbola at the bottom of the hippocampus in this plane. Using the plastic transfer pipette, gently squirt some ACSF into the gap 
underneath the fimbria to help separate the hippocampus from the cortex. Gently slide the spatula into this gap, such that the long side of the spatula runs parallel with the long axis of the hippocampus. Once the spatula is completely under the hippocampus, hold down the brainstem/ midbrain/thalamus firmly with the scissors and roll the spatula away from your body to physically separate the hippocampus from the rest of the brain. Once the hippocampus is free, gently trim away any remaining cortex, blood vessels, and white matter. Scoop a small amount of ACSF slushy onto the far side of the dissecting stage. Gently position the hippocampus next to the slush and douse with a few milliliters of ACSF using the plastic transfer pipette. Next, remove the other hemisphere and repeat the dissection.

*You may need the scissors to snip away additional connective tissue, white matter, or vasculature that prevents separation of the cortex from the brainstem. The points of your scissors will be very close to the hippocampus, so be sure to deliver very precise snips (sharp scissors are a must).

\section{Brain Removal and Hippocampal Dissection in Aged Transgenic Mice}

1. Euthanize and decapitate the mouse and make a midline incision on the scalp as described in section 2.3. Mice have much thinner skulls than rats which greatly simplifies brain extraction. Cutting through the skull with scissors is therefore unnecessary. Use bone rongeurs with smaller jaws (Table 2) to pull away occipital, parietal, and temporal skull plates. As with the rat, remember to use controlled movements and firmly pull the lower jaw of the rongeurs into the internal surface of the skull and away from the brain as you remove the plates. Once the plates are removed, use the narrow end of the Hippocampal tool to sever remaining cranial nerves and scoop the brain into ice-cold $\mathrm{Ca}^{2+}{ }^{2}$ free ACSF.

2. Prepare brain slices. Due to the smaller size of the mouse brain, dissecting the hippocampi can be a bit more challenging (though certainly doable) than when using rats. So, to make things easier, we remove the cerebellum and rostral tips of the frontal lobes, but do not dissect out the hippocampi. Instead, brain hemispheres are physically separated with a scalpel blade and left intact for Vibratome sectioning (see Section 4 below).

\section{Section Brain Tissue Into Slices using a Vibrating Microtome (Vibratome) and Transfer to Holding Chamber*}

1. Fill the reservoir of the Vibratome with ice-cold $\mathrm{Ca}^{2+}$-free ACSF, such that the cutting stage and blade are completely submerged. For making rat slices, cut off the rostral and caudal tips of each hippocampus with a scalpel blade. These cuts will allow you to vertically position the hippocampi closely together like two columns. This works best if the dentate gyrus of each hippocampus is facing one another and CA3 regions are oriented in the same direction. For making mouse slices, vertically position each hemisphere closely together with frontal lobes facing down. Glue brain tissue onto a mounting block and transfer to the sectioning stage of the Vibratome. We typically prepare $\sim 400$ uM sections for synaptic physiology experiments. Thinner sections should be prepared if fluorescent imaging will be conducted (e.g. investigations of $\mathrm{Ca}^{2+}$ levels and transients). Collect slices with a wide-mouth pipette or a paint brush\# and transfer to a small petri dish containing ice-cold $\mathrm{Ca}^{2+}$-free ACSF.

*Use of a Vibratome minimizes damage to the upper and lower surfaces of the slice and is definitely recommended for studies requiring analysis of cells near the slice surface (e.g. voltage clamp and $\mathrm{Ca}^{2+}$ imaging studies). However, cheaper alternatives are available and suitable for generating slices for extracellular physiology experiments. We have used a small gravity-controlled chopper, ${ }^{2,11}$ and a Mcllwain Tissue Chopper ${ }^{12}$ with good success. For this procedure hippocampi are placed on a staged and sectioned with a vertical chopper. A brush is used to transfer slices (one at a time) to a small holding dish filled with ice-cold $\mathrm{Ca}^{2+}$-free ACSF. One problem with this approach is that the hippocampi can move between chops resulting in uneven sections. Also, be careful to remove as much white matter, and especially as much vasculature, as possible before chopping. This material will stick to the brush, the razor blade, or both, making slice transfer very difficult and increasing the probability of stretching or damaging the tissue.

\#When using a brush, try to rest the slice length-wise across the bristles. Wrapping the slice around the brush tip may cause unnecessary tissue stretching.

2. Transfer brain slices to the holding chamber where they are bathed in oxygenated $\mathrm{Ca}^{2+}$-containing ACSF. Gradually bring the chamber temperature from $27^{\circ}$ to $32^{\circ} \mathrm{C}\left(+1^{\circ}\right.$ every five minutes). Allow slices to incubate for $1-1.5 \mathrm{~h}$ before electrophysiologic experiments.

\section{Elicit and Record CA3-CA1 Synaptic Responses}

1. For basic extracellular recordings in acute slices, your electrophysiology station will need to include*: a recording chamber; a perfusion system; a microscope with > 4X magnification capability; recording, stimulating, and ground electrodes; macro and micromanipulators; a rigid vibration-resistant table-top and Faraday cage; a stimulator; amplifier and analog-to-digital (A/D) converter; oscilloscope (preferably); and personal PC with appropriate acquisition software.

${ }^{*}$ Kerr Scientific Instruments offers a fantastic and inexpensive electrophysiology system (i.e. the Kerr Tissue Recording System) for conducting a variety of basic slice electrophysiology experiments. This system has a small footprint, portable stimulators and amplifiers, and can be used on a standard lab-bench without the need of a bulky Faraday cage.

Of course, brain slices can also be used for performing numerous elaborate electrophysiologic and imaging techniques, which will require additional equipment and materials. For instance, our main electrophysiology station contains an amplifier with fast voltage- and currentclamp capabilities, a fluorescent illumination system, and a digital camera. This station is used for extracellular field recordings in brain slices, as well as patch-clamp recordings and fluorescent imaging in slices and cell cultures ${ }^{3,12,13}$. See Table 3 for a full listing of equipment and components.

2. Using a wide-mouth pipette or small paint brush, transfer one or more slices to the recording chamber* allowing it to acclimate for 10-15 min before stimulation/recording. For our studies, slices are submerged in ACSF and rest on netting inserted into an RC-22 chamber by Warner Instruments (see Figure 3). ACSF is gravity-fed through a regulator to adjust flow-rate, and prewarmed to $32^{\circ} \mathrm{C}$ by an inline micro-heater before reaching the recording chamber. A central vacuum line is used to remove ASCF. 
*Many different varieties of submersible and interface-style recording chambers are commercially available. We have observed that slices exhibit more robust synaptic responses in an interface chamber (i.e. slice sits at an interface with humidified air and ACSF) ${ }^{2,11}$. However, responsivity is generally more stable when slices are submerged in ACSF. Drug perfusion is also more efficient in submersion chambers.

3. Position stimulating and recording electrodes. With the stimulator or stimulus isolator turned on, but output dialed down to 0 , position a stimulating electrode over the slice in the stratum radiatum region of CA2 near the CA3 border (see Figure 4). We use a twisted platinum iridium wire to deliver 50-100 uS diphasic pulses to CA3 Schaffer collateral (SC) fibers. The use of platinum iridium wire and diphasic pulses can help minimized electrode polarization. Lower a recording electrode into CA1 stratum radiatum, just breaking the surface of the slice. Our recording electrode is a $\mathrm{Ag} / \mathrm{AgCl}$ wire in an ACSF-filled glass micropipette (tip resistance of $\sim 7 \mathrm{M} \Omega$ ). Turn the output on the stimulator up to a moderate level (we set our stimulus isolator to $\sim 150 \mu \mathrm{A}$ ) and begin administering stimulus pulses and acquiring activity using acquisition software, such as Clampex from Axon Instruments, Inc. Slowly lower the stimulating electrode in small intervals until a stimulus artificact is recorded in CA1. Next, continue to slowly lower both the stimulating and recording electrodes in intervals while acquiring CA1 responses until the fiber volley (FV) and the excitatory postsynaptic potential (EPSP) amplitudes reach maximal levels (see Figure 4B).

4. Establish a synaptic strength curve and investigate synaptic plasticity. To generate a synaptic strength curve, deliver stimulus pulses to SC at increasingly higher intensities and record the corresponding activity in CA1. The range and number of stimulus intensity levels used may vary but should be sufficient to generate a sigmoidal curve when plotted against either FV or EPSP values (Figure 5). The FV amplitude provides a reliable estimate of the proportion of presynaptic fibers activated, while the EPSP slope provides an uncontaminated measure of monosynaptic CA3-CA1 currents. Plotting the EPSP slope against the FV across stimulus intensity levels therefore reflects the size of the EPSP per number of activated afferents and provides an excellent estimate of CA3-CA1 synaptic strength. Typically, synaptic strength levels are significantly reduced in area CA1 of aged rats and APP/PS1 mice, relative to young rats and age-matched wild-type mice e.g. see 4,14.

5. Slices that show signs of poor health (i.e. a maximal EPSP amplitude $<1 \mathrm{mV}$ ) or hyperexcitability (i.e. the appearance of 2 or more population spikes) during the synaptic strength curve are excluded from statistical analysis (see Figure 4C). We have found that these slices rarely exhibit stable responses across a $60 \mathrm{~min}$ period and/or show highly variable responses following induction of synaptic plasticity. It deserves noting that "unhealthy slices" make up only about $10-20 \%$ of all slices transferred to the recording chamber. Moreover, in our experience, the frequency of identifying an unhealthy slice is very similar across age, species, and genotype.

6. Induce long-term potentiation (LTP) or long-term depression (LTD) in slices. LTP and LTD are lasting increases (LTP) and decreases (LTD) in synaptic function in response to different patterns of synaptic activation. Both processes are widely believed to reflect critical mechanisms for learning and memory 15 and provide useful outcome measures for investigating cellular mechanisms of neural dysfunction and/or for assessing pharmacologic strategies for ameliorating memory deficits and neurodegeneration ${ }^{16}$.

For synaptic plasticity experiments, reset the stimulus intensity for all slices to $1 \mathrm{mV}^{*}$ and begin baseline stimulation at a frequency of 0.033 $\mathrm{Hz}$. EPSP slope values should be stable for at least 20 min prior to the induction of LTP or LTD. Closely monitor EPSPs during this time and reset the stimulus intensity if the slope fluctuates more than $10 \%$ and start a new baseline. Induce LTP using a one second train of $100 \mathrm{~Hz}$ stimulation or multiple short bursts ( 10 pulses) of $100 \mathrm{~Hz}$ stimulation given every $200 \mathrm{~ms}$. For LTD induction, deliver 900 stimulus pulses at a rate of $1 \mathrm{~Hz}$. After induction of LTP or LTD, collect synaptic responses for an additional 60 min or more. EPSP slope values obtained before and $60 \mathrm{~min}$ after high/low frequency stimulation are compared to determine the presence of LTP or LTD.

Aged rats and APP/PS1 mice tend to show deficient LTP and enhanced LTD (see Figure 5), and these changes have been suggested to contribute to impaired cognition in these animal models ${ }^{6,16}$. However, unlike APP/PS1 mice, changes in LTP/LTD in aged rats are variable across labs. Aged rats generally exhibit similar LTP levels compared to adults in response to "intense" (i.e. $100 \mathrm{~Hz}$ ) stimulation, but show deficits when milder stimulation parameters are used (i.e. lower stimulus frequencies or fewer stimulus pulses) (for review see ${ }^{4,6,16}$ ). Also, some labs, including ours, have observed increased susceptibility to LTD induction in aged rats ${ }^{2,17-19}$, while other groups have found no difference or reduced susceptibility in their aged animals. As discussed briefly below (see Discussion), subtle but critical differences in experimental protocol may account for these discrepancies.

*The stimulation intensity and EPSP amplitude can influence LTP induction and may be an important cause of discrepant results in the literature. This will be considered further in the Discussion section.

\section{Representative Results}

Our work, and work from other groups, suggests that changes in astrocyte-based inflammatory signaling may trigger and/or hasten neurologic dysfunction during aging and $A D^{13,20,21}$. Recently, we have used synaptic strength, LTP, and LTD as endpoint measures to investigate the efficacy and mechanisms of action of several novel anti-inflammatory reagents in mid-aged APP/PS1 mice (see ${ }^{22}$ for description of this model) and aged Fischer 344 rats. The results provided below were obtained using the protocols described in this article.

One of the novel anti-inflammatory adeno-associated viral (AAV) reagents developed by our lab has been shown in pilot studies to significantly increase synaptic strength $(p<0.05)$ and prevent LTP deficits $(p<0.05)$ in mid-aged (16-month-old) APP/PS1 mice $(n=4-6$ slices per treatment condition). Representative synaptic strength curves and LTP experiments from two different slices, collected from the same 16-month-old APP/PS1 mouse, are shown in Figure 5A-C. One slice was extracted from the hemisphere treated with our novel AAV (Reagent A), while the other slice was treated with a control AAV reagent (Control). LTP was induced in both slices using two 1 sec trains of $100 \mathrm{~Hz}$ stimulation (10 sec intertrain interval). Note that the synaptic strength curve for the Reagent A-treated slice is shifted to the left of the control slice, indicative of greater synaptic strength. Also note that, typical for mid-aged APP/PS1 mice, LTP decayed rapidly to baseline in the control slice (e.g. ${ }^{23}$ ). Conversely, LTP decayed little in the slice treated with our novel reagent.

In a second recent study, we observed significant LTD in vehicle-treated aged rats (85\% of pre-LTD baseline, $p<0.05)$. In contrast, no LTD was observed in aged rats treated with novel anti-inflammatory "Drug A" (97 \% of pre-LTD baseline, not significant). No drug effects on synaptic strength were observed. Representative LTD experiments from this data set ( $n=8-10$ rats per group) are illustrated in Figure 5D-F. 


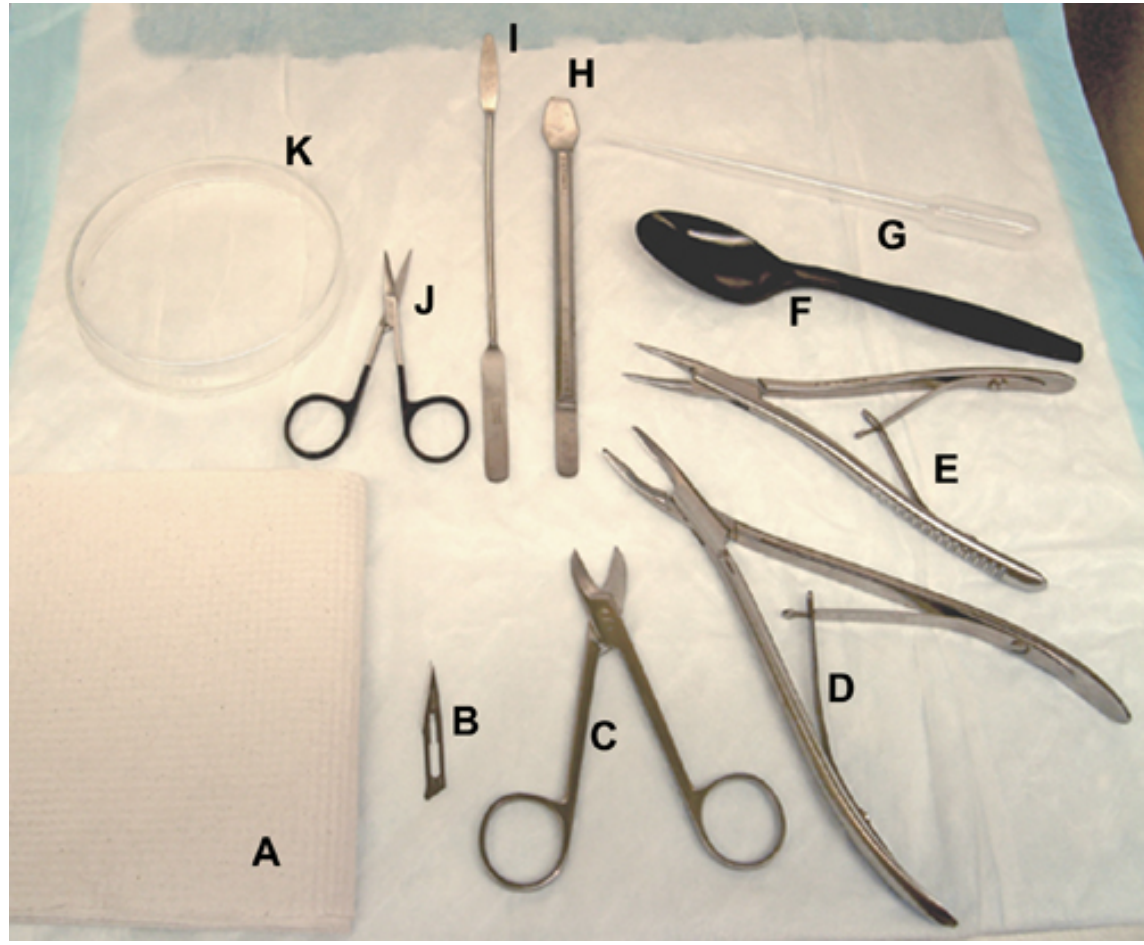

Figure 1. Tools and materials used for brain dissection. A, paper towel. B, scalpel blade. C, Beebee scissors. D, bone rongeurs (for rats). E, bone rongeurs (for mice/rats). F, Plastic spoon. G, plastic transfer pipette. H, Hippocampal tool. I, spatula. J, surgical scissors. K, glass petri dish.

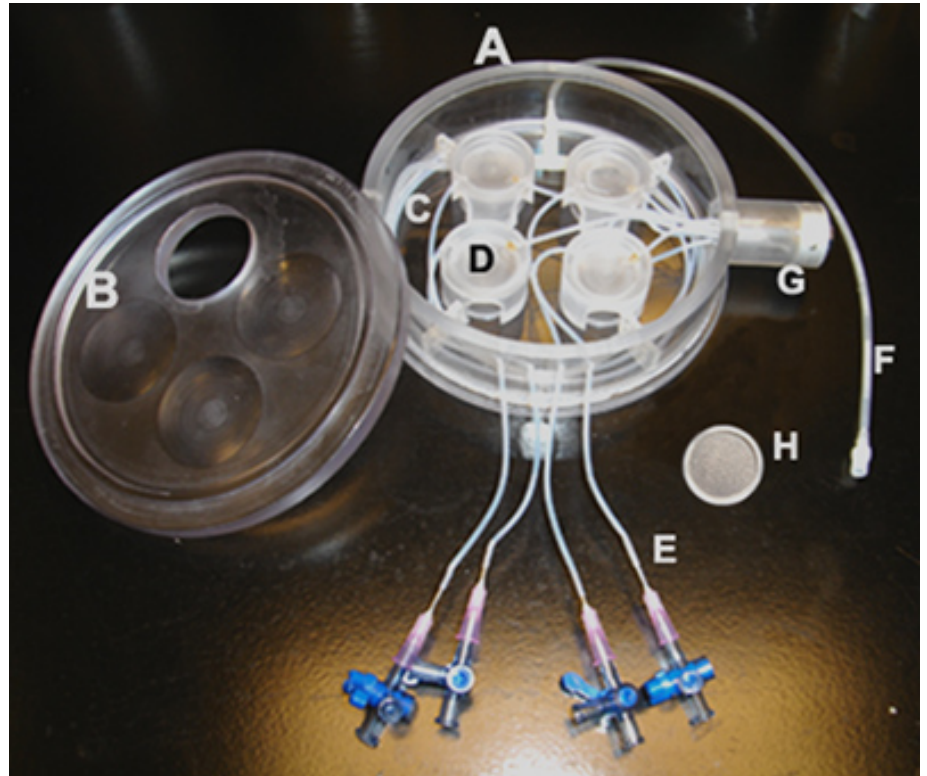

Figure 2. Custom brain slice holding chamber. A, macrochamber. B, lid. C, $\mathrm{H}_{2} \mathrm{O}$ reservoir with perforated silicone tubing. D, microchamber. $\mathrm{E}$, ACSF delivery tube (polyethylene). $\mathrm{F}, \mathrm{O}_{2}$ delivery tube. $\mathrm{G}$, port for temperature control. $\mathrm{H}$, netted microchamber insert. 


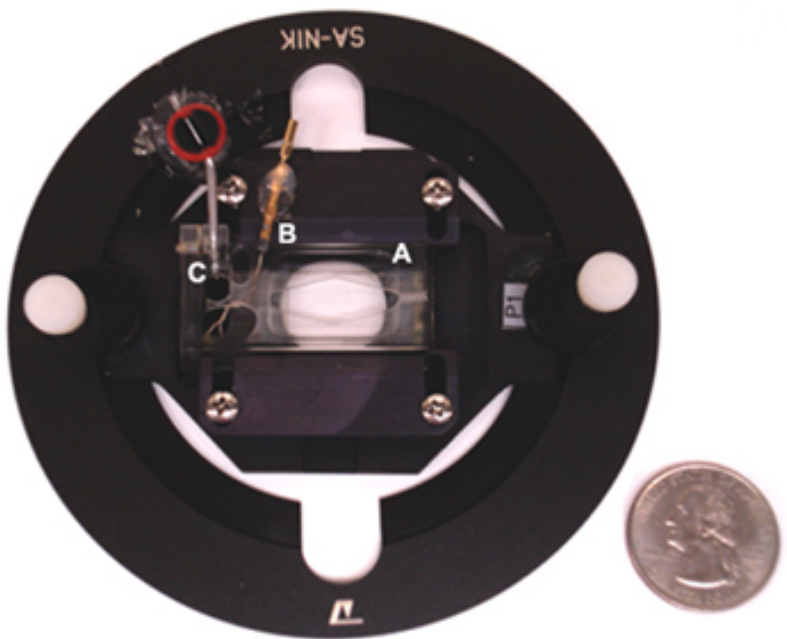

Figure 3. RC22 submersion chamber. A, Recording chamber. B, Ground electrode. C, Aspiration needle.
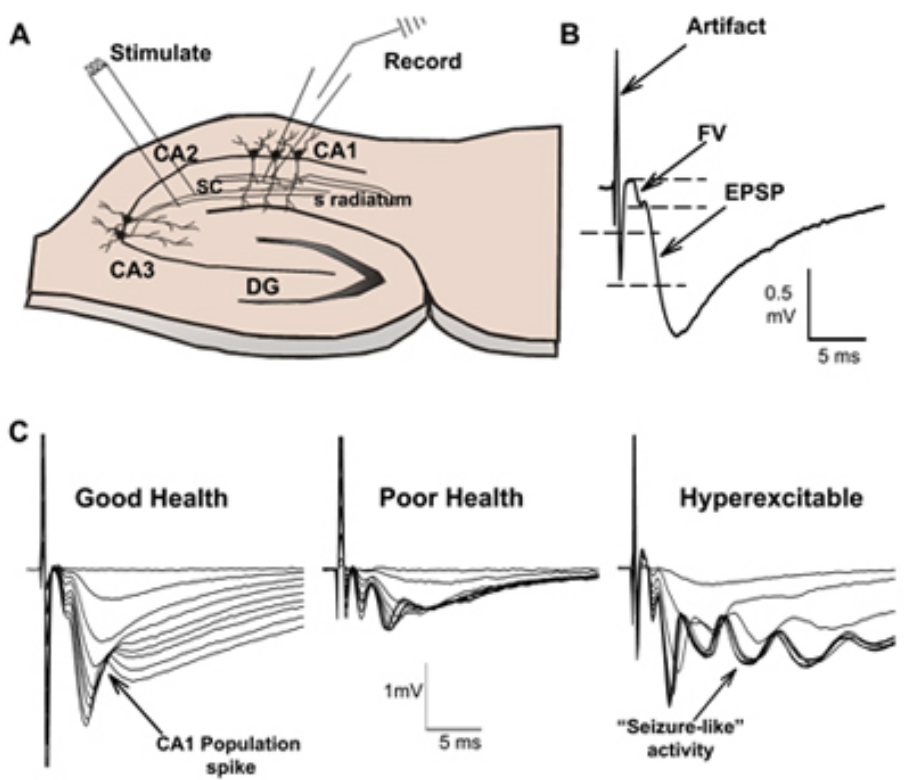

Figure 4. Hippocampal slice illustration and extracellular waveforms. A, Cartoon of a transverse hippocampal section used in electrophysiology experiments. $\mathrm{CA}=$ cornu Ammonis. $\mathrm{DG}=$ dentate gyrus. $\mathrm{SC}=$ Schaffer collaterals. $\mathrm{S}$ radiatum $=$ stratum radiatum. $\mathrm{B}$, Electrical stimulation of the SC (a CA3 axon tract) elicits a stimulus artifact, followed almost immediately by a presynaptic population spike, or fiber volley (FV). The amplitude of FV is directly proportional to the number of SC fibers activated. The slope of the negative-going phase of the field excitatory postsynaptic potential (EPSP) corresponds directly to the activation of depolarizing synaptic currents in CA1 pyramidal neurons in response to glutamate release from SC terminals. C, Overlapping representative extracellular waveforms recorded in CA1 stratum radiatum in response to nine different stimulus intensity levels (30-500 $\mu \mathrm{A})$ in a "healthy" (left panel), "unhealthy", and "hyperexcitable" slice. Five waveforms were averaged per level. Healthy slices respond dynamically across this stimulus range and exhibit a single positive-going population spike (reflecting CA1 neuronal discharge) at the higher stimulus levels. In the RC22 submersion chamber, maximal EPSPs typically range from 1.5 to $3 \mathrm{mV}$ in amplitude. Unhealthy slices (middle panel) often exhibit a large FV, but a small maximal EPSP $(<1 \mathrm{mV})$ and usually show poor plasticity. Hyperexcitable slices (right panel) show two or more regenerative population spikes in the ascending limb of the EPSP. Responses in hyperexcitable slices are often labile and are variably affected by LTD/LTP stimulation. 
A

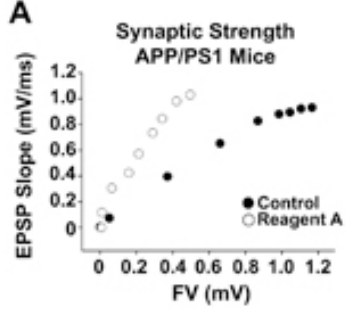

D

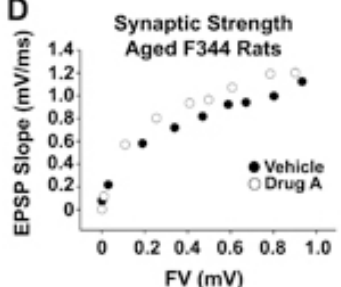

B

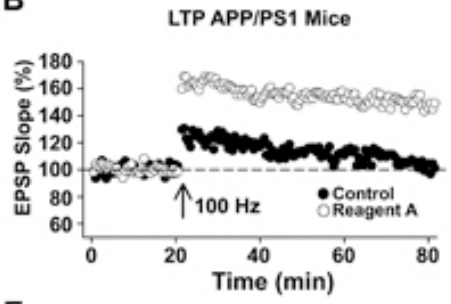

E

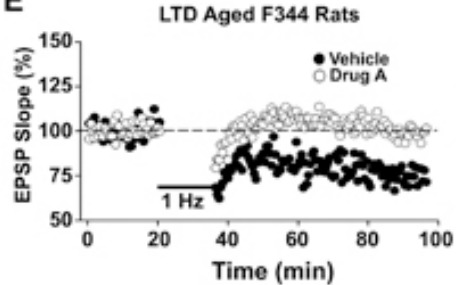

C

LTP APP/PS1 Mice

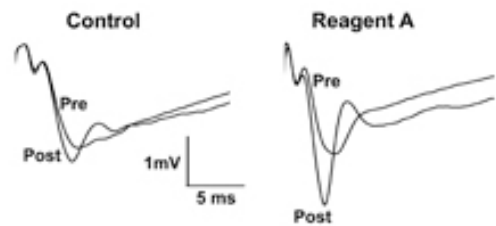

F

LTD Aged F344 Rats

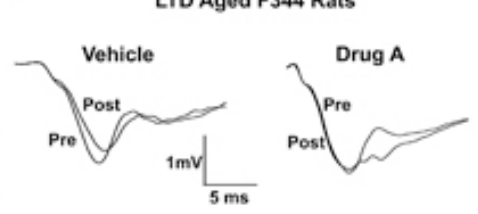

Figure 5. Representative electrophysiology experiments performed on acute slices from mid-aged (16 mos) APP/PS1 mice and aged (22 mos) Fisher 344 rats. Panels A-B show data collected from APP/PS1 mice treated with a control adeno-associated (AAV) viral construct (Control) or a novel AAV reagent (Reagent A) that has been developed by our lab group. Relative to the control slice, the slice treated with Reagent $A$ exhibits a marked leftward shift in the EPSP:FV curve $(A)$ indicative of greater synaptic strength. The Reagent-A-treated slice also shows robust and stable LTP (B) after the delivery of two $1 \mathrm{sec}, 100 \mathrm{~Hz}$ stimulus trains, while the control slice exhibits deficient LTP, typical of this animal model. Panels D-F show data collected from two individual aged rats that received chronic (4 week) intrahippocampal perfusions of vehicle or a novel anti-inflammatory drug (Drug A). Basal synaptic strength was relatively unaffected by drug treatment (D). However, Drug A was very effective at preventing the induction of $L T D(E)$. Panels $C$ and $F$ show representative EPSP waveforms recorded from individual slices before (pre) and 60 min after (post) the delivery of LTP/LTD stimulation. Note that stimulus artifacts are not shown.

\section{Discussion}

The steps outlined in this protocol will help insure that brain dissections are carried out at least as rapidly and efficiently in aged, as in young adult rats. We also provide sufficient detail for the beginner to set up their own slice studies on LTP and LTD. If further exploration of aging and $A D$ changes in synaptic function and plasticity is one of your goals, there are at least two other methodological issues, alluded to above, that deserve further consideration. First, several labs have shown that the $\mathrm{Ca}^{2+}: \mathrm{Mg} 2+$ ratio in recording ACSF can have a marked effect on the induction synaptic plasticity in hippocampal slices ${ }^{2,10,24,25}$. In mammalian CSF, the $\mathrm{Ca}^{2+}: \mathrm{Mg} 2+$ ratio is approximately one $\left(e . g\right.$. see ${ }^{26}$ ). However, ACSF Ca ${ }^{2+}: \mathrm{Mg} 2+$ ratios closer to 2 are commonly used in slice studies of synaptic function and plasticity. In early studies, this practice was probably adapted to optimize induction of LTP, then subsequently became routine for all plasticity studies. However, this practice can be problematic in aging and $A D$ studies because of well-characterized differences in neuronal $\mathrm{Ca}^{2+}$ regulation. Specifically, $\mathrm{Ca}^{2+}$ influx and/or $\mathrm{Ca}^{2+}$ induced $\mathrm{Ca}^{2+}$-release is elevated in aged rats and/or AD model mice during neuronal activation ${ }^{3,27-31}$. Induction of LTD is particularly sensitive to subtle changes in ACSF Ca ${ }^{2+}$ levels. Our protocol, which uses $2 \mathrm{mM} \mathrm{Ca}^{2+}$ and $2 \mathrm{mM} \mathrm{Mg} 2+$, commonly results in LTD for aged, but not young adult animals ${ }^{2}$, while studies using a $\mathrm{Ca}^{2+}: \mathrm{Mg} 2+$ ratio closer to two, have observed robust LTD in adults in the absence of an age difference ${ }^{2,10}$ or in conjunction with reduced LTD in aged rats ${ }^{32}$. These observations highlight the need to carefully consider $\mathrm{ACSF} \mathrm{Ca}^{2+}$ and Mg2+ levels when comparing $\mathrm{Ca}^{2+}$-dependent plasticity in aged and young adult animals.

The second methodological issue concerns the strong dependency of LTP on postsynaptic depolarization ${ }^{33}$ and possible aging/genotype differences in synaptic strength. In a typical LTP experiment, baseline and LTP stimulation intensity is generally adjusted to produce a half maximal (or three-quarter maximal) EPSP amplitude. The potential problem is that aged rats and APP/PS1 mice usually show reduced synaptic strength relative to their younger and/or wild type counterparts, meaning that baseline EPSP values will also be smaller in aged rats and APP/ PS1 mice. Smaller EPSPs may translate to less depolarization during LTP stimulation, resulting in a reduced probability for inducing LTP ${ }^{33}$. Because of this potential confound, it is difficult to determine whether these animals exhibit a throughput deficit, a plasticity deficit or both. That is, LTP induction mechanisms in aged and/or APP/PS1 mice may be functionally intact (no plasticity deficit), but insufficiently stimulated (throughput deficit) under these conditions. This distinction is critical, as mechanisms for throughput and mechanisms for plasticity may respond very differently to a specific pharmacologic treatment. We try to minimize the impact of reduced throughput on LTP induction by normalizing the EPSP amplitude to the same level (e.g. $1 \mathrm{mV}$ ) across all slices prior to LTP stimulation. Other strategies may be effective as well (e.g. use of voltage or current clamp to equalize the membrane potential across groups during LTP stimulation), and should be considered when investigating LTP in these animal models.

\section{Disclosures}

The production of this video-article was sponsored by Leica Microsystems.

\section{Acknowledgements}

Work supported by NIH grant AG027297, an award from the Kentucky Spinal Cord and Head Injury Research Trust, and a gift from the Kleberg Foundation. 


\section{References}

1. Norris, C. M., Halpain, S. \& Foster, T. C. Alterations in the balance of protein kinase/phosphatase activities parallel reduced synaptic strength during aging. J Neurophysiol 80, 1567-1570 (1998).

2. Norris, C. M., Korol, D. L. \& Foster, T. C. Increased susceptibility to induction of long-term depression and long-term potentiation reversal during aging. J Neurosci 16, 5382-5392 (1996).

3. Norris, C. M. et al. Hippocampal 'zipper' slice studies reveal a necessary role for calcineurin in the increased activity of L-type Ca(2+) channels with aging. Neurobiol Aging 31, 328-338, doi:S0197-4580(08)00107-3 [pii] 10.1016/j.neurobiolaging.2008.03.026 (2010).

4. Burke, S. N. \& Barnes, C. A. Senescent synapses and hippocampal circuit dynamics. Trends Neurosci 33, 153-161, doi:S0166-2236(09)00202-1 [pii] 10.1016/j.tins.2009.12.003 (2010).

5. Foster, T. C. Calcium homeostasis and modulation of synaptic plasticity in the aged brain. Aging Cell 6, 319-325, doi:ACE283 [pii] 10.1111/ j.1474-9726.2007.00283.x (2007).

6. Foster, T. C. \& Norris, C. M. Age-associated changes in $\mathrm{Ca}(2+)$-dependent processes: relation to hippocampal synaptic plasticity. Hippocampus 7, 602-612, doi:10.1002/(SICI)1098-1063(1997)7:6<602::AID-HIPO3>3.0.CO;2-G (1997).

7. Thibault, O., Gant, J. C. \& Landfield, P. W. Expansion of the calcium hypothesis of brain aging and Alzheimer's disease: minding the store. Aging Cell 6, 307-317, doi:ACE295 [pii] 10.1111/j.1474-9726.2007.00295.x (2007).

8. Demuro, A., Parker, I. \& Stutzmann, G. E. Calcium signaling and amyloid toxicity in Alzheimer disease. J Biol Chem 285, 12463-12468, doi:R109.080895 [pii] 10.1074/jbc.R109.080895 (2010).

9. Green, K. N. \& LaFerla, F. M. Linking calcium to Abeta and Alzheimer's disease. Neuron 59, 190-194, doi:S0896-6273(08)00586-2 [pii] 10.1016/j.neuron.2008.07.013 (2008).

10. Kumar, A., Thinschmidt, J. S., Foster, T. C. \& King, M. A. Aging effects on the limits and stability of long-term synaptic potentiation and depression in rat hippocampal area CA1. J Neurophysiol 98, 594-601, doi:00249.2007 [pii] 10.1152/jn.00249.2007 (2007).

11. Norris, C. M., Halpain, S. \& Foster, T. C. Reversal of age-related alterations in synaptic plasticity by blockade of L-type Ca ${ }^{2+}$ channels. J Neurosci 18, 3171-3179 (1998).

12. Norris, C. M. \& Scheff, S. W. Recovery of afferent function and synaptic strength in hippocampal CA1 following traumatic brain injury. J Neurotrauma 26, 2269-2278, doi:10.1089/neu.2009.1029 (2009).

13. Sama, M. A. et al. Interleukin-1 beta-dependent signaling between astrocytes and neurons depends critically on astrocytic calcineurin/NFAT activity. J Biol Chem 283, 21953-21964, doi:M800148200 [pii] 10.1074/jbc.M800148200 (2008).

14. Ye, H., Jalini, S., Mylvaganam, S. \& Carlen, P. Activation of large-conductance $\mathrm{Ca}(2+)$-activated $\mathrm{K}(+)$ channels depresses basal synaptic transmission in the hippocampal CA1 area in APP (swe/ind) TgCRND8 mice. Neurobiol Aging 31, 591-604, doi:S0197-4580(08)00165-6 [pii] 10.1016/j.neurobiolaging.2008.05.012 (2010).

15. Bear, M. F. \& Malenka, R. C. Synaptic plasticity: LTP and LTD. Curr Opin Neurobiol 4, 389-399 (1994).

16. Foster, T. C. Involvement of hippocampal synaptic plasticity in age-related memory decline. Brain Res Brain Res Rev 30, 236-249, doi:S016501739900017X [pii] (1999).

17. Foster, T. C. \& Kumar, A. Susceptibility to induction of long-term depression is associated with impaired memory in aged Fischer 344 rats. Neurobiol Learn Mem 87, 522-535, doi:S1074-7427(06)00184-5 [pii] 10.1016/j.nlm.2006.12.009 (2007).

18. Hsu, K. S. et al. Alterations in the balance of protein kinase and phosphatase activities and age-related impairments of synaptic transmission and long-term potentiation. Hippocampus 12, 787-802, doi:10.1002/hipo.10032 (2002).

19. Vouimba, R. M., Foy, M. R., Foy, J. G. \& Thompson, R. F. 17beta-estradiol suppresses expression of long-term depression in aged rats. Brain Res Bull 53, 783-787, doi:S0361923000003774 [pii] (2000).

20. Abdul, H. M., Furman, J. L., Sama, M. A., Mathis, D. M. \& Norris, C. M. NFATs and Alzheimer's Disease. Mol Cell Pharmacol 2, 7-14 (2010).

21. Abdul, H. M. et al. Cognitive decline in Alzheimer's disease is associated with selective changes in calcineurin/NFAT signaling. J Neurosci 29 , 12957-12969, doi:29/41/12957 [pii] 10.1523/JNEUROSCI.1064-09.2009 (2009).

22. Anantharaman, M. et al. Beta-amyloid mediated nitration of manganese superoxide dismutase: implication for oxidative stress in a APPNLH/ NLH X PS-1P264L/P264L double knock-in mouse model of Alzheimer's disease. Am J Pathol 168, 1608-1618, doi:168/5/1608 [pii] (2006).

23. Gengler, S., Hamilton, A. \& Holscher, C. Synaptic plasticity in the hippocampus of a APP/PS1 mouse model of Alzheimer's disease is impaired in old but not young mice. PLoS One 5, e9764, doi:10.1371/journal.pone.0009764 (2010).

24. Stringer, J. L. \& Lothman, E. W. In vitro effects of extracellular calcium concentrations on hippocampal pyramidal cell responses. Exp Neurol 101, 132-146, doi:0014-4886(88)90070-2 [pii] (1988).

25. Landfield, P. W., Pitler, T. A. \& Applegate, M. D. The effects of high Mg2+-to-Ca ${ }^{2+}$ ratios on frequency potentiation in hippocampal slices of young and aged rats. J Neurophysiol 56, 797-811 (1986).

26. Ames, A., $3^{\text {rd }}$, Sakanoue, M. \& Endo, S. Na, K, Ca, Mg, and C1 Concentrations in Choroid Plexus Fluid and Cisternal Fluid Compared with Plasma Ultrafiltrate. J Neurophysiol 27, 672-681 (1964).

27. Gant, J. C., Sama, M. M., Landfield, P. W. \& Thibault, O. Early and simultaneous emergence of multiple hippocampal biomarkers of aging is mediated by $\mathrm{Ca}^{2+}$-induced $\mathrm{Ca}^{2+}$ release. J Neurosci 26, 3482-3490, doi:26/13/3482 [pii] 10.1523/JNEUROSCI.4171-05.2006 (2006).

28. Thibault, O., Hadley, R. \& Landfield, P. W. Elevated postsynaptic $\left[\mathrm{Ca}^{2+}\right]$ i and L-type calcium channel activity in aged hippocampal neurons: relationship to impaired synaptic plasticity. J Neurosci 21, 9744-9756, doi:21/24/9744 [pii] (2001).

29. Thibault, O. \& Landfield, P. W. Increase in single L-type calcium channels in hippocampal neurons during aging. Science 272, $1017-1020$ (1996).

30. Stutzmann, G. E., Caccamo, A., LaFerla, F. M. \& Parker, I. Dysregulated IP3 signaling in cortical neurons of knock-in mice expressing an Alzheimer's-linked mutation in presenilin1 results in exaggerated $\mathrm{Ca}^{2+}$ signals and altered membrane excitability. J Neurosci 24, 508-513, doi:10.1523/JNEUROSCI.4386-03.2004 24/2/508 [pii] (2004).

31. Stutzmann, G. E. et al. Enhanced ryanodine receptor recruitment contributes to $\mathrm{Ca}^{2+}$ disruptions in young, adult, and aged Alzheimer's disease mice. J Neurosci 26, 5180-5189, doi:26/19/5180 [pii] 10.1523/JNEUROSCI.0739-06.2006 (2006).

32. Lee, H. K., Min, S. S., Gallagher, M. \& Kirkwood, A. NMDA receptor-independent long-term depression correlates with successful aging in rats. Nat Neurosci 8, 1657-1659, doi:nn1586 [pii] 10.1038/nn1586 (2005). 
33. McNaughton, B. L., Douglas, R. M. \& Goddard, G. V. Synaptic enhancement in fascia dentata: cooperativity among coactive afferents. Brain Res 157, 277-293, doi:0006-8993(78)90030-6 [pii] (1978). 